\title{
Hydrogen bonding in hybrid organic-inorganic perovskite materials
}

\author{
Xiaoping Wang \\ Neutron Scattering Division, Oak Ridge National Laboratory, Oak Ridge, TN 37831 USA \\ wangx@ornl.gov
}

Hybrid organic-inorganic perovskite (HOIP) materials have shown immense potential in high performance photovoltaics. However, significant challenges remain for real-world applications. A fundamental understanding of how the organic cations within inorganic framework affect the structural phase transitions, and optoelectronic properties of the HOIP materials is desirable to design new materials and improve device performance. We have used the TOPAZ instrument at the ORNL Spallation Neutron Source to probe the role of hydrogen bonding in structural phase transition of HOIPs by collecting the 3D volume of diffraction pattern from the sample in neutron event mode. The array of the TOPAZ neutron time-of-flight detectors covers a large 3D-volumes of Q-space (after unit conversion of each event data from detector $\mathrm{x}, \mathrm{y}$ and neutron wavelength in diffraction space) for highly efficient reciprocal space surveys. Connecting the timestamp of event data with that of external stimuli provides the tools needed to resample the multidimensional dataset for temporal filtering of event-based single crystal neutron diffraction data. This approach has opened a new avenue to probe structural phase transitions and dynamics in real time.

In this presentation, I will show the result from a real-time variable temperature study (3D in diffraction, 1D in temperature) that established the path of the organic cation induced anomalous optoelectronic phenomenon in MAPbX3, where MA is methylammonium, an organic cation that forms a network of hydrogen bonds with the halides X in the solid states. Data from real-time single-crystal neutron diffraction following the initiation of tetragonal-orthorhombic phase transition (Figure 1) provided details the change of hydrogen bonding pattern between the organic donor and the inorganic accepter. The change in hydrogen-bonding interaction not only induces the structural transition that results in anomalous red-shift of PL peak position as temperature increases, but also causes the decrease in dielectric screening, leading to the reduction of non-radiative recombination for stronger PL intensity.

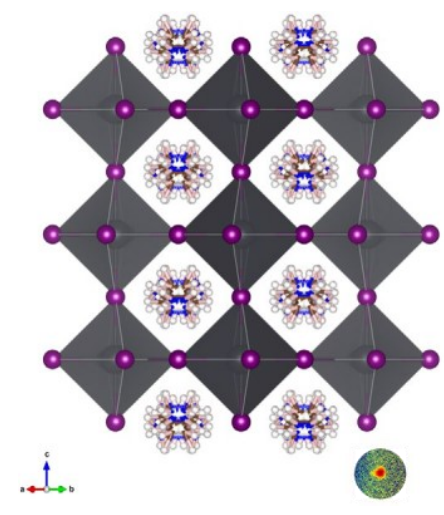

Tetragonal, $180 \mathrm{~K}$

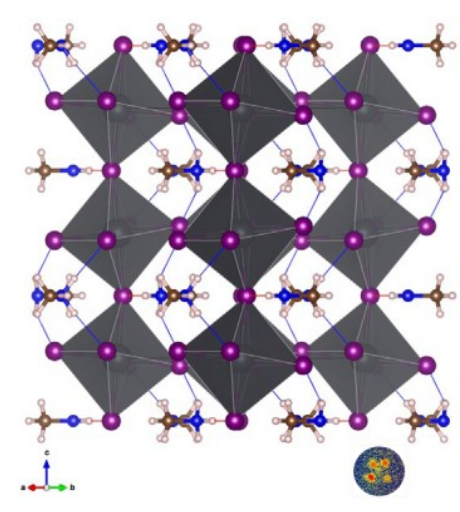

Orthorhombic, $95 \mathrm{~K}$

Figure 1. Hydrogen bonding and octhedral tilting of $\mathrm{MAPbBr}_{3}$. The insert shows the peak spliting at low $\mathrm{T}$.

\section{Keywords: hybrid organic-inorganic perovskite, neutron diffraction, hydrogen bonding, event data, multidimensional crystallography}

Acknowledgement: The single-crystal neutron diffraction experiment was performed at the TOPAZ beamline of Oak Ridge National Laboratory's Spallation Neutron Source, which is sponsored by the Scientific User Facilities Division, Office of Basic Energy Sciences, U.S. Department of Energy. 